Research, Society and Development, v. 11, n. 1, e8711124389, 2022

(CC BY 4.0) | ISSN 2525-3409 | DOI: http://dx.doi.org/10.33448/rsd-v11i1.24389

\title{
Influência do uso de psicoativos na autopercepção da memória: análise durante a pandemia de SARS-CoV-2
}

Influence of psychoactive use on self-perception of memory: an analysis during the SARS-CoV-2 pandemic

Influencia del consumo de drogas psicoactivas en la memoria autopercibida: análisis durante la pandemia de SARS-CoV-2

Recebido: 06/12/2021 | Revisado: 13/12/2021 | Aceito: 18/12/2021 | Publicado: 02/01/2022

Raphaela Barroso Guedes-Granzotti

ORCID: https://orcid.org/0000-0002-9064-439X Universidade Federal de Sergipe, Brasil E-mail: raphaelabgg@academico.ufs

Rodrigo Dornelas

ORCID: https://orcid.org/0000-0002-9710-5751

Universidade Federal do Rio de Janeiro, Brasil E-mail: rodrigodornelas@medicina.ufrj.br

Carla Patrícia Hernandez Alves Ribeiro César ORCID: https://orcid.org/0000-0002-9439-9352

Universidade Federal de Sergipe, Brasil E-mail: carlacesar@academico.ufs.br

Vanessa Veis Ribeiro

ORCID: https://orcid.org/0000-0002-3089-6085 Universidade de Brasília, Brasil E-mail: fgavanessavr@gmail.com Lucas Tito Pereira

ORCID: https://orcid.org/0000-0001-8235-079X Universidade Federal do Rio de Janeiro, Brasil E-mail: tito.lucas@gmail.com

Nathália Monteiro Santos ORCID: https://orcid.org/0000-0003-2746-5361 Universidade Federal de Sergipe, Brasil

E-mail: nathaliamonteiro.fono@gmail.com Kelly da Silva

ORCID: https://orcid.org/0000-0002-9193-7282 Universidade Federal de Sergipe, Brasil E-mail: kelly.silva@academico.ufs.br

\section{Resumo}

O presente estudo teve como objetivo verificar a influência do uso de psicoativos na autopercepção da memória retrospectiva e prospectiva durante a pandemia de SARS-CoV-2. Trata-se de um estudo transversal, observacional e analítico conduzido em 2020 com a população brasileira. A pesquisa foi realizada por meio do preenchimento de questionários digitais contendo perguntas referentes a dados sociodemográficos, conhecimentos relacionados à pandemia, uso e frequência de substâncias psicoativas e o protocolo Prospective and Retrospective Memory Questionnaire (PRMQ-10). Os resultados foram analisados por estatística descritiva e pela regressão linear múltipla, com um nível de significância de 5\%. Participaram 361 pessoas, sendo a maioria do gênero feminino, com ensino superior completo, que não pertenciam ao grupo de risco para a COVID-19 e que residiam com pessoas deste grupo. Quanto à frequência do uso de substâncias psicoativas, a maioria da amostra fez uso durante a pandemia de SARSCoV-2 de forma esporádica. A frequência de uso de remédios com e sem prescrição foi capaz de prever a pontuação total do PRMQ-10, evidenciando que o uso de substâncias psicoativas utilizados interferiram na autopercepção da memória retrospectiva e prospectiva.

Palavras-chave: COVID-19; Psicotrópicos; Memória; Pandemias.

\section{Abstract}

The present study aimed to verify the influence of psychoactive drug use on retrospective and prospective selfperception of memory during the SARS-CoV-2 pandemic. This is a cross-sectional, observational and analytical study conducted in 2020 with the Brazilian population. The research was carried out by filling out digital questionnaires containing questions related to sociodemographic data, knowledge related to the pandemic, use and frequency of 
Research, Society and Development, v. 11, n. 1, e8711124389, 2022

(CC BY 4.0) | ISSN 2525-3409 | DOI: http://dx.doi.org/10.33448/rsd-v11i1.24389

psychoactive substances and the Prospective and Retrospective Memory Questionnaire (PRMQ-10) protocol. The results were analyzed using descriptive statistics and multiple linear regression, with a significance level of 5\%. A total of 361 people participated, most females, with complete higher education, who did not belong to the group at risk for COVID-19 and who lived with people in this group. As for the frequency of use of psychoactive substances, most of the sample used it sporadically during the SARS-CoV-2 pandemic. The frequency of use of prescription and over-the-counter medications predicted the total PRMQ-10 score showing that the use of psychoactive substances used interfered with the self-perception of retrospective and prospective memory.

Keywords: COVID-19; Psychotropic Drugs; Memory; Pandemics.

\section{Resumen}

El presente estudio tuvo como objetivo verificar la influencia del uso de psicofármacos en la autopercepción de la memoria retrospectiva y prospectiva durante la pandemia del SARS-CoV-2. Se trata de un estudio transversal, observacional y analítico realizado en 2020 con población brasileña. La investigación se llevó a cabo mediante la cumplimentación de cuestionarios digitales que contienen preguntas relacionadas con datos sociodemográficos, conocimientos relacionados con la pandemia, uso y frecuencia de sustancias psicoactivas y lo protocolo del Prospective and Retrospective Memory Questionnaire (PRMQ-10). Los resultados se analizaron mediante estadística descriptiva y regresión lineal múltiple, con un nivel de significancia del 5\%. Participaron un total de 361 personas, en su mayoría mujeres, con estudios superiores completos, que no pertenecían al grupo de riesgo de COVID-19 y que convivían con personas de este grupo. En cuanto a la frecuencia de uso de sustancias psicoactivas, la mayor parte de la muestra se utilizó esporádicamente durante la pandemia del SARS-CoV-2. La frecuencia de uso de medicamentos con y sin prescripción fue capaz de predecir la puntuación total del PRMQ-10, mostrando que el uso de sustancias psicoactivas utilizadas interfirió en la autopercepción de la memoria retrospectiva y prospectiva.

Palabras clave: COVID-19; Psicotrópicos; Memoria; Pandemias.

\section{Introdução}

As substâncias psicoativas são aquelas que alteram a percepção das sensações, bem como a consciência e o estado emocional (Reuter \& Pardo, 2017). Tais substâncias podem ser organizadas em três tipos: 1) as depressoras do Sistema Nervoso Central (SNC), em que há diminuição da atenção, memória, tensão e cognição, como os ansiolíticos, álcool, inalantes e narcóticos; 2) as estimuladoras do SNC, em que há, por exemplo, a instauração de estado de euforia, aumento da vigília, da atenção e diminuição do apetite, como a cafeína, tabaco, anfetaminas, cocaína e o crack e; 3) as alucinógenas, que alteram as percepções, como o dietilamida do Ácido Lisérgico (LSD), o metilenodioximetanfetamina ou ecstasy, a cannabis sativa (maconha) e as substâncias derivadas de plantas ou cogumelos, como a ayahuasca, a ibogaína, a sálvia, a mescalina e a psilocibina (Silveira \& Doering-Silveira, 2014). O consumo de substâncias psicoativas tem sido considerado elevado e tem sido um problema crescente em alguns países, principalmente em virtude do surgimento de novas substâncias, que não constam na lista das Nações Unidas como controladas, evidenciando a necessidade de ampla discussão sobre o assunto (Negrei et al., 2017).

Os motivos para o uso dessas substâncias podem variar de acordo com a idade, o gênero e a condição social, sendo associado seu uso para relaxar, melhorar o desempenho em uma atividade, aliviar os sintomas de depressão e estresse e, até mesmo por experiências diárias de discriminação (Hadjiefthyvoulou et al., 2011; Solowij et al., 2011; Caqueo-Urízar et al., 2021). Um dos efeitos comuns ao uso de psicoativos é o déficit de memória, com alterações sendo observadas em todos os aspectos da função da memória, como codificação, armazenamento e recordação. Além disso, falhas de memória podem estar relacionadas a eventos e noções temporais, situações do futuro ou do passado, em que dificuldades na evocação de situações ocorridas no passado são denominadas falhas de memória retrospectiva, enquanto que dificuldades na evocação de intenções que devem ser realizadas no futuro, são denominadas de falhas de memória prospectiva (Wade et al., 2021; Blest-Hopley et al., 2021; Benites \& Gomes, 2007).

A pandemia de SARS-CoV-2 fez emergir uma crise sanitária e, sobretudo, política, cultural e econômica, pois trouxe à tona as desigualdades sociais entre diversas populações do mundo e instituiu modos de lidar com uma nova circulação social 
Research, Society and Development, v. 11, n. 1, e8711124389, 2022

(CC BY 4.0) | ISSN 2525-3409 | DOI: http://dx.doi.org/10.33448/rsd-v11i1.24389

(Fisher et al., 2020). Durante a pandemia percebeu-se o aumento do número de usuários de substâncias psicoativas (United Nations Office on Drugs and Crime, 2019), que pode ser justificado pelas sensações de medo e ansiedade na população (Ahorsu et al., 2020) e também pelo estresse pós-traumático, confusão e raiva que podem persistir a longo prazo (Brooks et al., 2020).

Assim, a adaptação para essa diferente realidade de confinamento, risco de contágio, crise financeira entre outros, pode desencadear distúrbios emocionais. Embora não haja um consenso quanto à forma de interação, estudos mostram que o consumo de alguns psicoativos está associado às situações estressoras, com o objetivo de trazer alívio (Chodkiewicz et al., 2020). Exposto isso, o referido estudo tem como objetivo verificar a influência do uso de psicoativos durante a pandemia de SARS-CoV-2 na autopercepção da memória retrospectiva e prospectiva de brasileiros.

\section{Metodologia}

Trata-se de um estudo observacional, transversal e analítico (Pereira et al., 2018). A pesquisa seguiu as recomendações éticas mundiais e nacionais para estudo com seres humanos e foi aprovada pelo Comitê de Ética em Pesquisa da Universidade Federal de Sergipe com o número de parecer 4012774 e CAAE 30092520.0.0000.5257. Todos os participantes assinaram de forma digital o Termo de Consentimento Livre e Esclarecido e receberam automaticamente uma via deste termo.

\section{Participantes}

Fizeram parte deste estudo 361 indivíduos que foram recrutados por meio de divulgação da pesquisa via e-mail, WhatsApp e por redes sociais do Brasil. A participação ocorreu de forma remota, por meio da plataforma digital Google Forms. Foram incluídos indivíduos com idades entre 18 e 60 anos, de qualquer gênero e residentes no Brasil. Foram excluídos os participantes que não preencheram todos os dados dos formulários.

\section{Material}

A coleta de dados foi realizada entre 29 de setembro e 24 de dezembro de 2020. Inicialmente os participantes responderam a um questionário elaborado pelos autores com questões sociodemográficas, aspectos relacionados à pandemia, e ao uso e frequência de substâncias psicoativas e, para avaliação da autopercepção da memória, o instrumento Prospective and Retrospective Memory Questionnaire-10 (PRMQ-10).

O questionário elaborado pelos autores foi composto por questões com informações referentes a gênero, escolaridade, ocupação, estado civil, renda familiar, pertencer ou residir com pessoas que fazem parte do grupo de risco para SARS-CoV-2, impacto da pandemia na renda familiar, e uso e frequência ("não usa ou/uso esporádico" ou "uso frequente") de substâncias psicoativas durante a pandemia (tabaco e derivados, como cigarros/charuto/cachimbo/fumo de corda; bebidas alcóolicas; cannabis sativa; cocaína; inalantes - lança perfume/loló/cola de sapateiro; remédio com e sem prescrição médica para ansiedade, tranquilizantes e remédios para dormir).

O questionário PRMQ-10, validado para o português brasileiro (Benites \& Gomes, 2007) apresenta 10 itens referentes às dificuldades com a memória no cotidiano, das quais cinco itens se referem à memória retrospectiva e os outros cinco, à memória prospectiva. As respostas são em escala Likert de acordo com à frequência de ocorrência, que vão de 1 (nunca) a 5 (quase sempre), sendo que para cada pergunta há uma pontuação possibilitando pontuação máxima de 50 pontos, indicando alta frequência de falhas de memória e pontuação mínima de 10 pontos, indicando baixa frequência de falhas de memória. 


\section{Análise dos dados}

Foi realizada de forma descritiva e inferencial. A descrição das variáveis qualitativas nominais foi realizada por meio de frequência relativa e absoluta. A descrição das variáveis quantitativas foi realizada por meio de medidas de variabilidade (desvio-padrão), tendência central (média e mediana) e posição (mínimo, máximo, primeiro quartil e terceiro quartil). Para análise inferencial dos resultados foi realizada uma regressão linear múltipla, com um nível de significância de 5\%.

\section{Resultados}

Os participantes da pesquisa apresentaram idade média de $31,1 \pm 10,7$ anos, com maior frequência do sexo feminino, indivíduos com Ensino Superior Completo, solteiros, com faixa de renda familiar compreendida entre um e três saláriosmínimos brasileiro. Em relação aos aspectos relacionados à pandemia, a maioria referiu não ter tido redução em sua renda familiar neste período, e embora a maioria não pertença ao grupo de risco para o agravamento da doença SARS-CoV-2, residiam com alguém que compõem este grupo. A Tabela 1 apresenta detalhadamente estes resultados.

Tabela 1. Perfil sociodemográfico e aspectos relacionados à pandemia por COVID-19 nos participantes da pesquisa.

\begin{tabular}{|c|c|c|}
\hline Variável & Frequência absoluta & Frequência relativa $(\%)$ \\
\hline \multicolumn{3}{|l|}{ Sexo } \\
\hline Masculino & 88 & 24,38 \\
\hline Feminino & 273 & 75,62 \\
\hline \multicolumn{3}{|l|}{ Escolaridade } \\
\hline Ensino Fundamental Completo & 1 & 0,28 \\
\hline Ensino Médio Incompleto & 2 & 0,55 \\
\hline Ensino Médio Completo & 22 & 6,09 \\
\hline Ensino Superior Incompleto & 122 & 33,80 \\
\hline Ensino Superior Completo & 214 & 59,28 \\
\hline \multicolumn{3}{|l|}{ Ocupação } \\
\hline Empregado & 152 & 42,11 \\
\hline Desempregado & 34 & 9,42 \\
\hline Aposentado & 1 & 0,28 \\
\hline Estudante & 174 & 48,20 \\
\hline \multicolumn{3}{|l|}{ Estado civil } \\
\hline Solteiro & 221 & 61,22 \\
\hline Casado & 94 & 26,04 \\
\hline União estável & 31 & 8,59 \\
\hline Divorciada & 14 & 3,88 \\
\hline Viúvo & 1 & 0,28 \\
\hline \multicolumn{3}{|l|}{ Faixa de renda* } \\
\hline$<1$ salário & 58 & 16,07 \\
\hline 1 a 3 salários & 116 & 32,13 \\
\hline 3 a 6 salários & 84 & 23,27 \\
\hline >6 salários & 103 & 28,53 \\
\hline \multicolumn{3}{|c|}{ Renda familiar diminuiu na pandemia } \\
\hline Não & 196 & 54,29 \\
\hline Sim & 165 & 45,71 \\
\hline
\end{tabular}


Grupo de risco para COVID-19

Não

Sim

Mora com pessoa do grupo de risco para a COVID-

19

Não

$\operatorname{Sim}$

Nota: *Um salário-mínimo brasileiro equivale hoje aproximadamente à $\mathrm{R} \$ 1.090,00$ reais, equivalente a $\$ 201,54$ dólares. Fonte: Autores.

A Tabela 2 apresenta a frequência de uso das substâncias psicoativas estudadas (variáveis independentes). Foi possível constatar que a maioria da amostra não utilizou substâncias psicoativa com frequência durante a pandemia.

Tabela 2. Frequência de uso das substâncias psicoativas.

\begin{tabular}{lcc}
\hline Variáveis independentes & Não uso ou uso esporádico & Uso frequente \\
$\mathbf{n}(\%)$ & $322(89,2)$ & $39(10,80)$ \\
\hline Derivados da nicotiana tabacum & $325(62,33)$ & $136(37,67)$ \\
Bebidas alcoólicas & $331(91,69)$ & $30(8,31)$ \\
Cannabis sativa & $312(86,43)$ & $49(13,57)$ \\
Remédios COM prescrição & $312(86,43)$ & $49(13,57)$ \\
Remédios SEM prescrição & $37)$ \\
\hline
\end{tabular}

Fonte: Autores.

A análise descritiva das variáveis dependentes estudadas com o protocolo PRMQ-10 está representada na Tabela 3.

Tabela 3. Análise descritivas das variáveis do estudo.

\begin{tabular}{|c|c|c|c|c|c|c|c|}
\hline Variáveis Independentes & Média & DP & Mínimo & Máximo & $1 Q$ & Mediana & $3 Q$ \\
\hline Idade & 31,13 & 10,67 & 18,00 & 59,00 & 22,00 & 27,00 & 38,00 \\
\hline Derivados da nicotiana tabacum & 0,34 & 0,86 & 0,00 & 3,00 & 0,00 & 0,00 & 0,00 \\
\hline Bebidas alcoólicas & 0,98 & 0,92 & 0,00 & 3,00 & 0,00 & 1,00 & 2,00 \\
\hline Cannabis sativa & 0,29 & 0,76 & 0,00 & 3,00 & 0,00 & 0,00 & 0,00 \\
\hline Cocaína & 0,00 & 0,00 & 0,00 & 0,00 & 0,00 & 0,00 & 0,00 \\
\hline Inalantes (lança perfume/loló/cola de sapateiro/ & 0,00 & 0,00 & 0,00 & 0,00 & 0,00 & 0,00 & 0,00 \\
\hline $\begin{array}{l}\text { Remédio COM prescrição para ansiedade, tranquilizantes } \\
\text { e remédios para dormir }\end{array}$ & 0,41 & 1,01 & 0,00 & 3,00 & 0,00 & 0,00 & 0,00 \\
\hline $\begin{array}{l}\text { Remédio SEM prescrição para ansiedade, tranquilizantes } \\
\text { e remédios para dormir }\end{array}$ & 0,36 & 0,91 & 0,00 & 3,00 & 0,00 & 0,00 & 0,00 \\
\hline \multicolumn{8}{|l|}{ Variável Dependente } \\
\hline \multicolumn{8}{|l|}{ Memória Prospectiva e Retrospectiva } \\
\hline PRMQ-10 Prospectiva & - & - & 5,00 & 24,00 & 11,00 & 13,00 & 16,00 \\
\hline PRMQ-10 Retrospectiva & - & - & 5,00 & 21,00 & 8,00 & 10,00 & 12,00 \\
\hline Pontuação total PRMQ-10 & - & - & 11,00 & 41,00 & 20,00 & 23,00 & 27,50 \\
\hline
\end{tabular}

Nota: PRMQ-10 - Prospective and Retrospective Memory Questionnaire. Fonte: Autores.

A regressão linear múltipla para verificar se a frequência de uso de substâncias psicoativas foi capaz de prever a pontuação total do PRMQ-10 resultou em um modelo estatisticamente significativo $[\mathrm{F}(2,358)=7,797 ; \mathrm{p}<0,001 ; \mathrm{R} 2=0,042]$. 
As variáveis independentes remédios (com e sem prescrição) foram previsores da pontuação total PRMQ-10, conforme pode ser observado na Tabela 4.

Tabela 4. Análise das substâncias psicoativas preditivas do resultado no questionário Prospective and Retrospective Memory Questionnaire (PRMQ-10).

\begin{tabular}{llllll}
\hline Modelo & B & Beta & T & p-valor & VIF \\
\hline (Constante) & 23,138 & & 65,755 & 0,000 & \\
Remédios COM prescrição (binário) & 3,072 & 0,178 & 3,433 & 0,001 & 1,000 \\
Remédios SEM prescrição (binário) & 1,792 & 0,104 & 2,004 & 0,046 & 1,000 \\
\hline
\end{tabular}

Fonte: Autores.

\section{Discussão}

A coleta de dados do presente estudo foi realizada em um momento que o Brasil era o terceiro país com maior número de casos acumulados de COVID-19 e o segundo com o maior número de óbitos pela doença, liderando o ranking dos países que compõem a América do Sul (Brasil, 2020), evidenciando as fragilidades em saúde daquele momento. A preocupação com o uso de substâncias psicoativas durante a pandemia está relacionada aos malefícios, que são de ordem abrangente e complexa, afetando a saúde e variáveis de ordem psicoemocional tanto do usuário quanto de sua rede social e familiar. Um estudo conduzido com 1145 brasileiros durante a pandemia de SARS-CoV-2 evidenciou que 32\% da amostra iniciou o consumo de psicoativos durante este período (Trinta Weber, 2020). Estes resultados levantam a discussão a respeito não apenas do consumo recreacional destas substâncias, mas também de sua implicação na vida das pessoas.

Na presente pesquisa, observou-se que a maioria da amostra não fez uso frequente de substâncias psicoativas. Entretanto, dentre as substâncias pesquisadas, o álcool apresentou-se como a mais consumida na categoria de uso frequente, assim como descrito por outro estudo nacional ((Trinta Weber, 2020). O álcool é uma das drogas mais utilizadas e embora os fatores ambientais e genéticos contribuam para a etiologia dos transtornos associados ao seu consumo, os impactos dessa substância no sistema nervoso justificam tanto as mudanças comportamentais agudas, como seu efeito estimulante sucedido por efeitos depressivos, quanto as mudanças crônicas no comportamento, incluindo uso escalonado, tolerância, busca compulsiva e dependência (Abrahao et al., 2017). O consumo de álcool não foi previsor da autopercepção da memória e da desvantagem vocal no presente estudo. Apesar disso, a preocupação pelo uso e abuso de álcool durante a atual Pandemia de SARS-CoV-2 dá-se também pela comprovada afetação das células pulmonares pelo álcool, colocando os seus usuários em um maior risco de gravidade se contaminados pela COVID-19 (Bailey et al., 2021).

Em segundo lugar o uso mais frequente foi de medicamentos, com e sem prescrição médica. Quem relatou utilizar medicamentos com prescrição médica, tais como ansiolíticos, tranquilizantes e indutores do sono, apresentou pior autopercepção da memória. No que diz respeito à comunicação humana e seus processos adjacentes, a memória de curto prazo, relacionada à retenção de informações e novos aprendizados pode ser prejudicada pelo uso de substâncias psicoativas (Volkow \& Baler, 2014).

Indivíduos que utilizam fármacos controlados sem prescrição médica apresentaram pior autopercepção da memória do que os que não utilizam. Acredita-se que isso tenha ocorrido porque os fármacos pesquisados se referem à ansiolíticos, tranquilizantes e indutores do sono, o que sugere que os consumidores destas substâncias apresentam sintomas de ansiedade e /ou problemas de sono que, independentemente do uso de psicoativos, podem interferir na memória (Moran, 2016). A discussão precisa ser ampliada, pois os benzodiazepínicos e as chamadas drogas $\mathrm{Z}$ têm sido amplamente utilizadas pela população mundial (em torno de 3\% da população) e seus efeitos em longo prazo não estão bem estabelecidos (Mander et al., 
2017). Portanto, se a prescrição destas substâncias realizada por médicos de forma indiscriminada representa um sério problema de saúde pública pelos seus efeitos adversos, a automedicação é um problema ainda mais sério e que exige medidas rígidas de fiscalização. O uso racional de medicamentos é uma política para conter o avanço da automedicação que pode ocasionar superdosagem ou efeitos não esperados pelo medicamento.

Drogas psicotrópicas como o lítio e a clozapina podem, clinicamente, simular os sintomas da COVID-19 (como a febre, a tosse, a dispneia e sinais digestivos) (Nader \& Gowing, 2020), requerendo cuidados especiais a fim de que sejam evitados falsos negativos ou positivos. Tais fármacos são utilizadas, na maioria dos casos, no intuito de diminuir a ansiedade, e sabe-se que houve aumentos dos casos de ansiedade durante a pandemia.

Em relação aos derivados da nicotiana tabacum, tem como seu princípio ativo a nicotina. Trata-se de uma substância que causa dependência química em até $80 \%$ dos casos. Seus efeitos podem ser estimulantes ou depressivos, e variam de acordo com a quantidade de cigarros consumida, o tempo de uso e a resposta orgânica (Javelot et al., 2020).

O PRQM foi utilizado em um estudo inglês associado ao Teste de Memória Prospectiva de Cambridge (CAMPROMPT), em fumantes e não fumantes. Não foram encontradas diferenças entre os grupos pelo PRQM, porém os fumantes apresentaram pior desempenho no CAMPROMPT, sugerindo que o tabagismo possa prejudicar na memória prospectiva cotidiana (Brasil, 2015). Esses resultados corroboram os achados do presente estudo em que a autopercepção da memória com o PRQM não diferenciou o uso frequente ou não de derivados da nicotiana tabacum.

Além disso, o tabaco é conhecido por seus efeitos como indutor da isoenzima docitocromo P450, família 1, subfamília A, polipeptídeo 2 -a CYP1A2(29). No momento em que um medicamento é administrado a um indivíduo, passa por biotransformação por meio de processos bioquímicos, sendo necessária a presença de enzimas para esse intento, sendo que a CYP1A2 participa da primeira fase desse metabolismo (Mander et al. 2017). Desta forma, em pacientes infectados pelo novo coronavírus, a interrupção do tabagismo deve ser indicada o mais rapidamente possível, principalmente na vigência de sintomas respiratórios (como a tosse e a dispneia) e para aqueles que fazem uso de psicotrópicos metabolizados pelo CYP1A2 (Javelot et al., 2020).

Em relação à cannabis sativa, foi a substância menos consumida dentre as pesquisadas. Ela tem o canabidiol como um dos princípios ativos, que diminui a expressão do medo e interrompe a reconsolidação da memória emocional do medo, aumentando a eliminação desse sentimento (Nader \& Gowing, 2020). Acredita-se que isso pode justificar o uso recreacional durante a pandemia em curso, que tem sido retratada como a pandemia do medo (Javelot et al., 2020). Um componente ativo da cannabis, o delta-9-tetra-hidrocanabinol (THC), que é o seu maior psicoativo, pode induzir sintomas psicóticos de forma aguda e comprometer fortemente as memórias episódica e de trabalho (Wade et al., 2021). Esse dado não se confirmou no presente estudo, o que pode estar relacionado ao baixo consumo dessa substância em relação as demais pesquisadas, visto que no Brasil ela é uma droga ilícita.

Diante dos dados expostos, o presente estudo apresentou como limitação o corte transversal no momento da pandemia, o que não permite comparar possíveis modificações de consumo antes e durante a pandemia.

\section{Conclusão}

Quanto à frequência do uso de substâncias psicoativas, a maioria da amostra fez uso durante a pandemia de SARSCoV-2 de forma esporádica. A frequência de uso de remédios com e sem prescrição foi capaz de prever a pontuação total do PRMQ-10, evidenciando que o uso de substâncias psicoativas utilizados interferiram na autopercepção da memória retrospectiva e prospectiva.

São necessários estudos caso-controle, bem como estudos com medidas objetivas para compreender melhor a 
Research, Society and Development, v. 11, n. 1, e8711124389, 2022

(CC BY 4.0) | ISSN 2525-3409 | DOI: http://dx.doi.org/10.33448/rsd-v11i1.24389

influência das substâncias psicoativas na memória.

\section{Referências}

Abrahao, K. P., Salinas, A. G., Lovinger, D. M. (2017). Alcohol and the brain: neuronal molecular targets, synapses, and circuits. Neuron, 96(6), 1223-38. https://doi.org/10.1016/j.neuron.2017.10.032.

Ahorsu, D. K., Lin, C. Y., Imani, V., Saffari, M., Griffiths, M. D., Pakpour, A.H. (2020). The fear of COVID-19 scale: development and initial validation. Int J ment Heal Addict, 27, 1-9. https://doi.org/10.1007/s11469-020-00270-8

Bailey, K. L., \& Samuelson, D. R., Wyatt, T. A. (2021). Alcohol use disorder: A pre-existing condition for COVID-19? Alcohol. 90, 11-17. https://doi.org/10.1016/j.alcohol.2020.10.003

Benites. D., \& Gomes, W. B. (2007). Tradução, adaptação e validação preliminar do Prospective and Retrospective Memory Questionnaire (PRMQ). Psico USF, 12(1):45-54. https://doi.org/10.1590/S1413-82712007000100006

Blest-Hopley, G., Giampietro, V., \& Bhattacharyya, S. (2020). A Systematic Review of Human Neuroimaging Evidence of Memory-Related Functional Alterations Associated with Cannabis Use Complemented with Preclinical and Human Evidence of Memory Performance Alterations. Brain Sciences, 10(2), 102. https://doi.org/10.3390/brainsci10020102

Brasil. (2020). Ministério da Saúde. Boletim epidemiológico especial: doença pelo coronavírus COVID-19. Ministério da Saúde, editor. Brasília.

Brasil. (2015). Ministério da Saúde. O cigarro brasileiro: análises e propostas para a redução do consumo. Rio de Janeiro: INCA.

Brooks, S. K., Webster, R. K., Smith, L. E., Woodland, L., Wessely, S., Greenberg, N., et al. (2020). The psychological impact of quarantine and how to reduce it: rapid review of the evidence. Lancet, 395(10227):912-20. http://dx.doi.org/10.1016/S0140-6736(20)30460-8

Caqueo-Urízar, A., Urzúa, A., Mena-Chamorro, P., Flores, J., Irarrázaval, M., Graniffo, E., \& Williams, D. R. (2021). Relationship between Everyday Discrimination and Substance Use among Adolescents in Northern Chile. International Journal of Environmental Research And Public Health, 18(12), 6485. https://doi.org/10.3390/ijerph18126485

Chodkiewicz, J., Talarowska, M., Miniszewska, J., Nawrocka, N., Bilinski, P. (2020). Alcohol consumption reported during the COVID-19 pandemic: The initial stage. Int J Environ Res Public Health, 17(13),1-11. https://doi.org/10.3390/ijerph17134677

Fisher, J., Languilaire, J.C., Lawthom, R., Nieuwenhuis, R., Petts, R.J., Runswick-Cole, K. et al. (2020). Community, work, and family in times of COVID-19. Community Work Fam, 23(3), 247-52. https://doi.org/10.1080/13668803.2020.1756568

Gigliotti, A., Ribeiro, M., Tapia Aguilera. A., Rezende, E., Ogata Perrenoud, L. (2014). Paradigms of public policies for licit and illicit drugs in Brazil. Subst Abus, 35(3), 292-7. https://doi.org/10.1080/08897077.2014.917351

Hadjiefthyvoulou, F., Fisk, J. E., Montgomery, C., Bridges, N. (2011). Prospective memory functioning among ecstasy/polydrug users: Evidence from the Cambridge Prospective Memory Test (CAMPROMPT). Psychopharmacology (Berl), 215(4), 761-74. https://doi.org/10.1007/s00213-011-2174-y

Javelot, H., Llorca, P-M., Drapier, D., Fakra, E., Hingray, C., Meyer, G. et al. (2020). Informations relatives aux psychotropes et à leurs adaptations éventuelles pour les patients souffrant de troubles psychiques en France pendant l'épidémie à SARS-CoV-2. Encephale, 46(3), S14-34. https://doi.org/10.1016/j.encep.2020.04.006

Mander, B. A., Winer, J. R. \& Walker, M. P. (2017). Sleep and Human Aging. Neuron, 94, 19-36. https://doi.org/10.1016/j.neuron.2017.02.004

Moran, T. P. (2016). Anxiety and working memory capacity: A meta-analysis and narrative review. Psychol Bull, 142(8), 831-64 https://doi.org/10.1037/bul0000051

Nader, D. \& Gowing, L. (2020). Is Long-Term Benzodiazepine Use a Risk Factor for Cognitive Decline? Results of a Systematic Review. J Addict, 1-10. https://doi.org/10.1155/2020/1569456

Negrei, C., Galateanu, B., Stan, M., Balalau, C., Dumitru, M. L. B., Ozcagli, E. et al. (2017). Worldwide legislative challenges related to psychoactive drugs. Daru 25(1), 14. https://doi.org/10.1186/s40199-017-0180-2

Pereira, A., Shitsuka, D., Parreira, F., \& Shitsuka, R. (2018). Metodologia da pesquisa científica. Núcleo de Tecnologia Educacional da Universidade Federal de Santa Maria

Reuter, P., Pardo, B. (2017). Can new psychoactive substances be regulated effectively? An assessment of the British Psychoactive Substances Bill, Addiction. 112(1), 25-31. https://doi.org/10.1111/add.13439

Silveira, D.X., Doering-Silveira, E. (2014). Classificação das substâncias psicoativas e seus efeitos. In: Brasil. Secretaria Nacional de Políticas sobre Drogas, editor. Prevenção dos problemas relacionados ao uso de drogas: capacitação para conselheiros e lideranças comunitárias. Brasília: SENAD-MJ/NUTE-UFSC, 66-86.

Snyderman, C., Weissmann, J., Tabor, E., Curtin, H. (1991). Crack Cocaine Burns of the Larynx. Arch Otolaryngol Neck Surg, 117(7), 792-5. https://doi.org/10.1001/archotol.1991.01870190104022

Solowij, N., Jones, K. A., Rozman, M. E., Davis, S. M., Ciarrochi, J., Heaven, P. C. L., et al. (2011). Verbal learning and memory in adolescent cannabis users, alcohol users and non-users. Psychopharmacology (Berl), 216(1),131-44. https://doi.org/10.1007/s00213-011-2203-x 
Research, Society and Development, v. 11, n. 1, e8711124389, 2022 (CC BY 4.0) | ISSN 2525-3409 | DOI: http://dx.doi.org/10.33448/rsd-v11i1.24389

Trinta Weber, C. A., Monteiro, I. T., Gehrke, J. M., Silva de Souza, W. (2020). The Use of Psychoactive Substances in the Context of the Covid-19 Pandemic in Brazil. medRxiv, https://doi.org/10.1101/2020.09.25.20194431

United Nations Office on Drugs and Crime (2019). The world drug report. United Nations publication. https://www.unodc.org/doc/wdr2016/WORLD_DRUG_REPORT_2016_web.pdf

Volkow, N. D., Baler, R. D., Compton, W. M. \& Weiss, S. R. B. (2014). Adverse Health Effects of Marijuana Use. N Engl J Med, 370(23), 2219-27. https://doi.org/10.1056/NEJMra1402309

Wade, N. E., Baca, R., Courtney, K. E., McCabe, C. J., Infante, M. A., Huestis, M. A., \& Jacobus, J. (2021). Preliminary Evidence for Cannabis and Nicotine Urinary Metabolites as Predictors of Verbal Memory Performance and Learning Among Young Adults. Journal of the International Neuropsychological Society, 27(6), 546-558. https://doi.org/10.1017/S1355617721000205 